OPEN ACCESS

Edited by: Enrico laccino, Magna Graecia University

of Catanzaro, Italy

Reviewed by:

Lavinia Raimondi,

Rizzoli Orthopedic Institute, Scientific Institute for Research, Hospitalization and Healthcare (IRCCS), Italy

Erika Cione,

University of Calabria, Italy Antonella Zannetti, Institute of Biostructure and Bioimaging, Consiglio Nazionale delle Ricerche (CNR), Italy

*Correspondence: Anne Blangy anne.blangy@crbm.cnrs.fr

Guillaume Bompard guillaume.bompard@crbm.cnrs.fr

${ }^{\dagger}$ These authors share senior authorship

Specialty section: This article was submitted to

Cellular Biochemistry,

a section of the journal

Frontiers in Cell and Developmental

Biology

Received: 17 September 2021

Accepted: 11 October 2021

Published: 18 November 2021

Citation:

Maurin J, Morel A, Guérit D,

Cau J, Urbach S, Blangy $A$ and Bompard G (2021) The Beta-Tubulin Isotype TUBB6 Controls Microtubule and Actin Dynamics in Osteoclasts.

Front. Cell Dev. Biol. 9:778887.

doi: 10.3389/fcell.2021.778887

\section{The Beta-Tubulin Isotype TUBB6 Controls Microtubule and Actin Dynamics in Osteoclasts}

\author{
Justine Maurin', Anne Morel1, David Guérit', Julien Cau², Serge Urbach ${ }^{3}$, \\ Anne Blangy ${ }^{1 *+}$ and Guillaume Bompard ${ }^{1 *+}$
}

\begin{abstract}
${ }^{1}$ Centre de Recherche de Biologie Cellulaire de Montpellier, CNRS, Montpellier University, Montpellier, France, ${ }^{2}$ BioCampus Montpellier, CNRS, INSERM, Montpellier University, Montpellier, France, ${ }^{3}$ Institute of Functional Genomics, CNRS, INSERM, Montpellier University, Montpellier, France
\end{abstract}

Osteoclasts are bone resorbing cells that participate in the maintenance of bone health. Pathological increase in osteoclast activity causes bone loss, eventually resulting in osteoporosis. Actin cytoskeleton of osteoclasts organizes into a belt of podosomes, which sustains the bone resorption apparatus and is maintained by microtubules. Better understanding of the molecular mechanisms regulating osteoclast cytoskeleton is key to understand the mechanisms of bone resorption, in particular to propose new strategies against osteoporosis. We reported recently that $\beta$-tubulin isotype TUBB6 is key for cytoskeleton organization in osteoclasts and for bone resorption. Here, using an osteoclast model CRISPR/Cas9 KO for Tubb6, we show that TUBB6 controls both microtubule and actin dynamics in osteoclasts. Osteoclasts $\mathrm{KO}$ for Tubb6 have reduced microtubule growth speed with longer growth life time, higher levels of acetylation, and smaller EB1-caps. On the other hand, lack of TUBB6 increases podosome life time while the belt of podosomes is destabilized. Finally, we performed proteomic analyses of osteoclast microtubule-associated protein enriched fractions. This highlighted ARHGAP10 as a new microtubule-associated protein, which binding to microtubules appears to be negatively regulated by TUBB6. ARHGAP10 is a negative regulator of CDC42 activity, which participates in actin organization in osteoclasts. Our results suggest that TUBB6 plays a key role in the control of microtubule and actin cytoskeleton dynamics in osteoclasts. Moreover, by controlling ARHGAP10 association with microtubules, TUBB6 may participate in the local control of CDC42 activity to ensure efficient bone resorption.

Keywords: osteoclast, tubulin isotype, microtubule, actin, bone resorption, GRAF2, PS-GAP

\section{INTRODUCTION}

Osteoclasts are giant multinucleated cells involved in bone resorption. Their counterparts, osteoblasts, participate in new bone formation. Both cell types are thus crucial for bone homeostasis which is highly dynamic despite bone stiffness. Furthermore, whereas an equilibrium between the activities of osteoclasts and osteoblasts is crucial for bone renewal, a pathological excess in osteoclast 
activity results in bone loss and may lead to osteoporosis; this is the case in number of physio-pathological situations such as from age-related sexual hormone decay, chronic inflammation, and cancer (Khosla and Hofbauer, 2017). Bone resorption by osteoclasts relies on their ability to organize a specific adhesive structure called the sealing zone or podosome belt, composed of actin rich $\alpha \mathrm{v} \beta 3$ integrin-containing adhesive structures called podosomes (Blangy et al., 2020). The sealing zone surrounds a highly convoluted plasma membrane domain: the ruffled border, which is specifically involved in bone resorption being the siege of acid and protease secretions respectively dissolving mineral matrix and digesting bone proteins. The dynamic of this podosomal structure allows osteoclast sliding and generates series of resorption pits at the bone surface (Søe and Delaissé, 2017).

Various approaches to regulate osteoclast activity have been developed, mostly focused on pathways regulating differentiation, but targeting the sealing zone formation also appears an interesting alternative for the treatment of osteoporosis (Blangy et al., 2020; Mounier et al., 2020). Combining transcriptomic and proteomic approaches, we recently highlighted the $\beta$-tubulin isotype TUBB6 as important for the establishment and/or the maintenance of the podosome belt and for osteoclast activity (Guérit et al., 2020). Microtubules are dynamic polymers of $\alpha$ - and $\beta$ - tubulin heterodimers, which oscillate between growing and shrinking phases, a process known as dynamic instability. The specific regulation of MT dynamics and organization allows their adaptation to diverse cellular functions, such as cell division, motility, shape, and intracellular transport. Tubulin isotypes and post-translational modifications generate a so-called "tubulin code" translated into a given function through the recruitment of specific microtubule-associated proteins (Janke and Magiera, 2020). Indeed, the nature of $\beta$-tubulin isotypes was shown to affect microtubule dynamics in vitro (Vemu et al., 2017; Ti et al., 2018). TUBB6 was found to exert a microtubule-destabilizing effect in cycling cells (Bhattacharya and Cabral, 2009; Bhattacharya et al., 2011). Moreover, TUBB6 is expressed at low levels in most tissues (Leandro-García et al., 2010), and high expression of TUBB6 is characteristic of osteoclasts (Guérit et al., 2020). Microtubules and the actin cytoskeleton are physically and functionally intimately connected (Dogterom and Koenderink, 2019). In particular in osteoclasts, both actin cytoskeleton and microtubules are key for the formation of the podosome belt and for bone resorption (Okumura et al., 2006), but their regulatory crosstalk remains poorly understood in osteoclast (Blangy et al., 2020).

We reported recently that TUBB6 plays a key role in actin organization in osteoclasts and their resorption activity (Guérit et al., 2020). In the present study, we investigated further how TUBB6 regulates actin cytoskeleton in osteoclast, through the development of a cellular model knocked out for Tubb6 gene. We found that actin organization and bone resorption defects of Tubb6 KO osteoclasts are correlated not only with changes in microtubule but also actin dynamics. Furthermore, we developed a proteomic approach and identified proteins whose association with microtubules is affected by TUBB6.

\section{MATERIALS AND METHODS}

\section{Mice, Cell Lines, and Lentivirus Production}

Four-week-old C57Bl/6J mice were purchased from Harlan France and maintained in the animal facilities of the CNRS in Montpellier, France. Procedures involving mice were performed in compliance with local animal welfare laws, guidelines, and policies, according to the rules of the regional ethical committee. RAW264.7 cells were a gift from Kevin P McHugh (Gainesville, FL, United States). Lentiviral particles were produced at the Plateforme de Vectorologie de Montpellier (Montpellier, France).

\section{Osteoclast Differentiation and Activity}

For osteoclast differentiation, RAW264.7 cells between passages 5 and 12 were cultured in a humidified incubator at $37^{\circ} \mathrm{C}$ and $5 \% \mathrm{CO}_{2}$, in $\alpha$-MEM supplemented with $10 \%$ heat inactivated fetal calf serum (Biowest), Penicillin-Streptomycin (Life Technologies), $2 \mathrm{mM}$ glutamine (Lonza), and $50 \mathrm{ng} / \mathrm{mL}$ RANKL (Peprotech). Medium was changed every other day until osteoclast differentiation at day 4 or 5 . When appropriated, at day 2 of differentiation, siRNAs were transfected with siImporter (Millipore) in OptiMEM medium (Life Technologies) containing $50 \mathrm{ng} / \mathrm{mL}$ RANKL, as described previously (Touaitahuata et al., 2016), using $100 \mathrm{nM}$ of the siRNAs described previously: luciferase control and Tubb6 siRNAs si1077 and si1373 (Guérit et al., 2020). Primary osteoclasts were obtained from mouse bone marrow as described previously (Vives et al., 2011; Touaitahuata et al., 2016). Briefly, non-adherent bone marrow cells from long bones of 6- to 8-week-old C57BL/6J mice were grown for $48 \mathrm{~h}$ in $\alpha$-MEM containing $10 \%$ heatinactivated fetal calf serum, $2 \mathrm{mM}$ glutamine, and $30 \mathrm{ng} / \mathrm{mL}$ macrophage colony-stimulating factor (M-CSF; Miltenyi) to obtain bone marrow macrophages (BMMs); BMMs were then grown in the same medium complemented with $50 \mathrm{ng} / \mathrm{mL}$ RANKL for 5 days to obtain osteoclasts. Differentiation was performed on glass or on Apatite Collagen Complex (ACC)-coated coverslips, prepared as reported previously (Maurin et al., 2018).

To measure their activity, osteoclasts were detached from the culture plate by scrapping at day 3 of differentiation and seeded for 3 more days onto inorganic crystalline calcium phosphate (CaP)-coated multiwells (Osteo Assay Surface, Corning), eight wells per condition. For each condition, 4 wells were then stained for tartrate resistant acid phosphatase (TRAP) activity to measure osteoclast surface and 4 wells stained with von Kossa to measure CaP dissolution as described previously (Brazier et al., 2009). Wells were imaged with a Nikon SMZ1000 stereomicroscope equipped with a Nikon DXM 1200F CCD camera and the quantifications were carried out with ImageJ $1.53 \mathrm{c}$ software. In each experiment, osteoclast specific activity was expressed as the average area resorbed in the four wells stained with von Kossa normalized by the osteoclast surface in the four wells stained with TRAP. 


\section{Generation of Tubb6 Knock-Out RAW264.7 Cells}

A guide RNA (gRNA) targeting the second exon of mouse Tubb6 gene ( $5^{\prime}$-CGACCAGGCCGGAGGCTACG-3') was designed and cloned in lentiCRISPRv2 vector, which also provides the expression of the Streptococcus pyogenes Cas9 and puromycin resistance, a gift from Feng Zhang (Addgene plasmid \# 52961; http://n2t.net/addgene:52961; RRID:Addgene_52961) (Sanjana et al., 2014). Empty and Tubb6 gRNA-containing lentiCRISPRv2 were used to produce lentiviruses and generate respectively control (WT) and Tubb6 KO RAW264.7 cells. For this, growing RAW264.7 cells were infected with lentiviral particles and selected with $3 \mu \mathrm{g} / \mathrm{ml}$ puromycin $48 \mathrm{~h}$ later. Puromycinresistant WT and Tubb6 KO RAW264.7 clones were individually picked, expanded, and TUBB6 expression was monitored by immunoblot analysis using home-made polyclonal antibodies against the C-terminal end of mouse TUBB6, generated in rabbit at Eurogentec by injection of peptide GGGEINE as described (Spano and Frankfurter, 2010). Selected clones of RAW264.7 cells were used to differentiate WT and Tubb6 KO osteoclasts.

\section{Quantitative Polymerase Chain Reaction}

DNaseI-treated total RNA was prepared using the RNeasy Minikit (Qiagen); RNA was primed with 10-mer random primers and reverse transcription was catalyzed using Superscript II reverse transcriptase (Invitrogen) to generate cDNAs. Quantitative Polymerase Chain Reaction (Q-PCR) was performed an Mx3000p PCR system (Stratagene) using the Platinium Taq DNA polymerase (Invitrogen) and SYBR Green I (Bio Wittaker) as described (Maurin et al., 2018). Primers used were 5'-ACAGTCCATGCCATCACTGCC-3' and 5'-GCCTGCTTCACCACCTTCTT-3' for Gapdh and $5^{\prime}$-GAACTATGTGCACCGGGACC-3' and 5'-GAGCTCGCTC AGCAGAATCC-3' for Src and $5^{\prime}$-TGGAGGCCTCTCTTG GTGTC- $3^{\prime}$ and CCACAAGATTCTGGGGACTC-3' for Cathepsin K. The threshold cycle $(\mathrm{Ct})$ of each amplification curve was calculated by Roche Diagnostics LightCycler 480 software using the second derivative maximum method. The relative amount of a given mRNA was calculated using the $\Delta \mathrm{Ct}$ method (Livak and Schmittgen, 2001).

\section{Immunoblot}

Osteoclast lysates were prepared in lysis buffer $(50 \mathrm{mM}$ Tris pH7.4, $150 \mathrm{mM} \mathrm{NaCl}, 1 \%$ IGEPAL CA-630, 2 mM EDTA, $50 \mathrm{mM} \mathrm{NaF}, 10 \%$ glycerol, protease inhibitor cocktail). Culture supernatants were collected to assess the secretion of Cathepsin K. Protein concentrations were determined with Bradford. After resolution by SDS-PAGE, proteins were transferred to PVDF-FL membranes (Immobilon-FL Transfer Membrane) and analyzed by immunoblot. Primary antibodies were: TUBB6 (1:1000), ARHGAP10 (ProteinTech S5136AP, 1:1000), GAPDH (CellSignaling \#2118, 1:5000), Sigma antibodies EB1 (E3406, 1:2500), K40-acetylated tubulin (T6793, 1:1000) and actin (A2103, 1:1000), Abcam antibodies Vinculin (ab108620, 1:5000), CLASP1 (ab108620, 1:5000) and Histone H3 (ab1791, 1:1000), total $\beta$ tubulin (Developmental Studies Hybridoma Bank
E7, 1:1000), Santa Cruz Biotechnologies antibodies TUBB5 (SAO.4G5, sc-58884, 1:5000) and Cathepsin K (E-7, sc-48353, 1:500). Secondary antibodies were: Goat-anti-mouse Dylight680 (Invitrogen, 35518), Goat-anti-mouse Dylight800 (Invitrogen, SA535521), Goat-anti-rabbit Dylight680 (Invitrogen, 35568), Goat-anti-rabbit Dylight800 (Invitrogen, SA535571). Signals were acquired using the Odyssey Infrared Imaging System (LICOR Biosciences, United States).

\section{Immunofluorescence}

All imaging was performed at Montpellier Ressources Imagerie (MRI) in Montpellier. For immunofluorescence, osteoclasts were differentiated on glass coverslips. Alternatively, osteoclasts at day 3 of differentiation were detached by scrapping and seeded for 2 more days on ACC as described previously (Maurin et al., 2018). Cells were fixed for $5 \mathrm{~min}$ in methanol at $-20^{\circ} \mathrm{C}$ followed by $10 \mathrm{~min}$ permeabilization in $0.1 \%$ Triton $\mathrm{X}-100$ in PBS in the case of endogenous EB1 labeling. Alternatively, cells were fixed for $20 \mathrm{~min}$ at room temperature in $3.2 \%$ paraformaldehyde and $10 \mu \mathrm{M}$ Paclitaxel (Sigma) in PHEM (60 mM PIPES, 25 mM HEPES, 10 mM EGTA, $4 \mathrm{mM} \mathrm{MgSO}_{4}$, $\mathrm{pH}$ 6.9) and cells were then permeabilized with $0.1 \%$ Triton $\mathrm{X}$ 100 in PBS for $1 \mathrm{~min}$. After blocking for $1 \mathrm{~h}$ with $2 \%$ BSA in PBS, cells were processed for immunofluorescence. Primary antibodies used were: TUBB6 (1:1000), $\alpha$-tubulin (1:2000, Sigma T5168) or $\alpha$-tubulin (1:200, YOL3/4 Santa Cruz Biotechnologies sc53030), ARHGAP10 (1:200), EB1 (BD 610534, 1:500) in 2\% BSA in PBS. Secondary antibodies used were from Invitrogen: donkey anti-mouse Alexa fluor 488 (Invitrogen A21202), donkey anti-rabbit Alexa fluor 546 (Invitrogen, A10040), goat anti-rat Alexa fluor 633 (Invitrogen, A21094), all used 1:1000. When appropriate, F-actin was labeled with Phalloidin Alexa fluor 647 (1:1000, Invitrogen A22287) or Phalloidin rhodamine (Sigma P1951, 1:10,000). Coverslips were mounted in Citifluor MWL488-25 (CliniSciences), imaged with Leica SP5 SMD confocal microscope equipped with oil objective 63X HCX Plan Apo CS oil 1.4NA under Metamorph 7.7.6.

\section{Image Quantification and Live Imaging}

Actin organization was evaluated visually after F-actin staining in fixed osteoclasts; a podosome belt was classified abnormal when F-actin staining was fragmented, weak, or absent in more than half of the osteoclast periphery, as described previously (Maurin et al., 2018).

For live imaging, osteoclasts were differentiated from WT and Tubb6 KO RAW264.7 cells expressing either LifeAct-mCherry to follow actin dynamics or EB1-GFP to follow microtubule dynamics. LifeAct-mCherry was subcloned into pMXs-Puro, retroviral particles were produced as described (Guimbal et al., 2019), and RAW264.7 cells were infected for $24 \mathrm{~h}$ in $8 \mu \mathrm{g} / \mathrm{ml}$ polybrene (Sigma). Alternatively, RAW264.7 were infected with lentiviral particles obtained from EB1-GFP lentiviral vector, a gift from Ken-Ichi Takemaru (Addgene plasmid \# 118084; RRID:Addgene_118084) ${ }^{1}$ for $5 \mathrm{~h}$ in $8 \mu \mathrm{g} / \mathrm{ml}$ polybrene (Sigma).

\footnotetext{
${ }^{1}$ http://n2t.net/addgene: 118084
} 
For microtubule regrowth assays, osteoclasts were treated with $10 \mu \mathrm{M}$ nocodazole on ice for $2.5 \mathrm{~h}$ to depolymerize microtubules. Medium was then replaced by nocodazole-free medium at $37^{\circ} \mathrm{C}$. At desired time points, cells were rinsed in PHEM containing $0.1 \%$ saponin, $0.25 \mathrm{nM}$ nocodazole, and $0.25 \mathrm{nM}$ paclitaxel, and fixed with methanol at $-20^{\circ} \mathrm{C}$ for $5 \mathrm{~min}$. After 1 -h saturation in $2 \%$ BSA in PBS, cells were processed for immunofluorescence to label microtubules with $\alpha$ tubulin antibody (1:2000) and donkey anti-mouse Alexa488 secondary antibody (1:1000). Images were acquired as described in the Immunofluorescence section above. Aster areas were measured using Segmented Line function in ImageJ $1.53 \mathrm{c}$.

For microtubule dynamics, EB1-GFP-expressing osteoclasts were imaged at $37^{\circ} \mathrm{C}$ with inverted spinning disk Nikon Ti Andor CSU-X1 equipped with EMCCD iXon897 Andor camera, one image per second for $2 \mathrm{~min}$, with oil objective $100 \mathrm{x}$ Plan Apo lambda 1.45 NA. EBI-GFP comet mean growth speed, mean length, and mean lifetime were determined using u-track (V2.0) software. Comet detection settings were 2 and 4 pixels respectively for low-pass and high-pass Gaussian standard deviation. For tracking the maximum gap to close was set to 4 frames while the minimum length of track segment from first step was set to 10 frames. The remaining settings used for tracking were the $\mathrm{u}$-track default ones. Images were also analyzed in Image J $1.53 \mathrm{c}$ with MTrackJ function microtubule growth speed, as described (Guimbal et al., 2019).

Endogenous EB1 and EB1-GFP comet length were measured with the Straight-Line function of ImageJ 1.53c.

For podosome belt dynamics, LifeAct-mCherry-expressing osteoclasts were imaged at $37^{\circ} \mathrm{C}$ with Dragonfly spinning disk microscope equipped with an EMCCD iXon888 Life Andor camera (Andor Technology), two images per minute, with 40X plan fluor 1,3 NA DT $0.2 \mu \mathrm{m}$ objective. The overlap of actin ring position between different time points was determined with the function Measure in ImageJ 1.53c.

For individual podosome dynamics, LifeAct-mCherryexpressing osteoclasts were imaged at $37^{\circ} \mathrm{C}$ with CSU-X1 spinning disk microscope as above, one image per second was acquired for $10 \mathrm{~min}$, with $100 \mathrm{X}$ plan Apo lambda 1.45 NA objective. Podosome life time was determined by tracking manually on the videos the interval between the appearance and disappearance of individual podosomes.

\section{Preparation of Osteoclast Microtubule-Associated Protein Enriched Fractions}

Osteoclasts were lysed in Tub/MT microtubule stabilizing buffer (80 mM MES, 1 mM EGTA, 4M glycerol, $2 \mathrm{mM} \mathrm{MgCl}_{2}, 1 \mathrm{mM}$ $\mathrm{Na}_{3} \mathrm{VO}_{4}, \mathrm{pH}$ 6.8) supplemented with $0.1 \%$ Triton $\mathrm{X}-100$ and protease inhibitory cocktail for $4 \mathrm{~min}$ at room temperature. Lysates were centrifuged for $10 \mathrm{~min}$ at room temperature at $16,000 \times g$. Pellets were resuspended in Tub/MT supplemented with $20 \mu \mathrm{M}$ paclitaxel, $0.1 \mu \mathrm{M}$ GTP, $350 \mathrm{mM} \mathrm{NaCl}$ and protease inhibitory cocktail and centrifuged at $21,500 \times g$ for $10 \mathrm{~min}$. The resulting supernatant $\mathrm{S} 2$, enriched in microtubule binding proteins, was further analyzed by proteomics.

\section{Microtubule Co-sedimentation Assay}

Human embryonic kidney 293T (HEK293T) cells were transfected with either pRK5 HA-GST (Bompard et al., 2018) or pRK5 HA-GST ARHGAP10, PCR-cloned into pRK5 HA-GST using pEGFP-N3 ARHGAP10 as template, a generous gift from Dr. Daisuke Mori (Sekiguchi et al., 2020), using jetPEI (Polyplus transfection) following manufacturer instructions. Twenty-four hours post transfection, cells were harvested, washed twice in ice cold 1X PBS, and lysed in 1X BRB80 (80 mM PIPES, $1 \mathrm{mM}$ EGTA, $1 \mathrm{mM} \mathrm{MgCl}$, pH 6.8) supplemented with $1 \mathrm{mM} \mathrm{DTT}$, protease inhibitor cocktail, and 0.1\% IGEPAL CA-630 for $20 \mathrm{~min}$ on ice. Cell lysates were clarified at $100,000 \times g$. for $30 \mathrm{~min}$ at $4^{\circ} \mathrm{C}$. $100 \mu \mathrm{g}$ of clarified lysates were supplemented with $2 \mathrm{mM}$ GTP, incubated $5 \mathrm{~min}$ on ice followed by $10 \mathrm{~min}$ at $37^{\circ} \mathrm{C}$. A total of $20 \mu \mathrm{M}$ paclitaxel was added and the reaction incubated at $37^{\circ} \mathrm{C}$ for 10 more min. Reaction was loaded on a $40 \%$ glycerol cushion made in 1X BRB80 supplemented with $20 \mu \mathrm{M}$ paclitaxel and centrifuged at $100,000 \times g$ for $15 \mathrm{~min}$ at $30^{\circ} \mathrm{C}$. Pellet was rinsed once with $1 \mathrm{X}$ BRB80 supplemented with $20 \mu \mathrm{M}$ paclitaxel and resuspended in the same volume as supernatant. For control experiments, all steps were performed at $4^{\circ} \mathrm{C}$ in the absence of GTP and Paclitaxel. Samples were then analyzed by immunoblot.

\section{Proteomics}

After reduction (DTT $1 \mathrm{M}, 30 \mathrm{~min}$ at $60^{\circ} \mathrm{C}$ ) an alkylation (IAA $0.5 \mathrm{M}, 30 \mathrm{~min}$ RT) microtubule-associated protein enriched fractions were digested using trypsin (Gold, Promega, $1 \mu \mathrm{g} /$ sample, overnight at $30^{\circ} \mathrm{C}$ ). Peptide clean-up was done using OMIX C18 (Agilent) according to manufacturer protocol. For LC MSMS analysis, samples were loaded onto a $25 \mathrm{~cm}$ reversed-phase column (75 $\mathrm{mm}$ inner diameter; Acclaim PepMap 100 C18; Thermo Fisher Scientific) and separated with an UltiMate 3000 RSLC system (Thermo Fisher Scientific) coupled to a QExactive HFX system (Thermo Fisher Scientific). Tandem mass spectrometry analyses were performed in a datadependent mode. Full scans $(350-1,500 \mathrm{~m} / \mathrm{z})$ were acquired in the Orbitrap mass analyzer with a resolution of 120,000 at $200 \mathrm{~m} / \mathrm{z}$. For MS scans, $3 \mathrm{e} 6$ ions were accumulated within a maximum injection time of $60 \mathrm{~ms}$. The 20 most intense ions with charge states $\geq 2$ were sequentially isolated (1e5) with a maximum injection time of $50 \mathrm{~ms}$ and fragmented by higher-energy collisional dissociation (normalized collision energy of 28) and detected in the Orbitrap analyzer at a resolution of 30,000. Raw spectra were processed with MaxQuant v 1.6.10.43 (Cox and Mann, 2008) using standard parameters with LFQ and match between runs option. Spectra were matched against the UniProt reference proteome of Mus musculus (UP000000589, release $2020 \_04^{2}$ ) and 250 frequently observed contaminants, as well as reversed sequences of all entries. The maximum false discovery rate for peptides and proteins was set to 0.01 . Representative protein ID in each protein group was automatically selected using the in-house developed Leading tool v 3.4 (Raynaud et al., 2018). Statistical analysis ( $t$-test) were done using Perseus v 1.6.10.43 (Tyanova et al., 2016). Briefly, after reverse and contaminants entries removal, LFQ data were $\log 2$ transformed. Imputation

\footnotetext{
${ }^{2}$ http://www.uniprot.org
} 
was performed using "Replace missing values from normal distribution" with standard parameters. Difference between the two groups was then assessed using a standard $t$-test.

GO term enrichment analyses were performed using Panther 16.0 software $^{3}$ (GO Ontology database DOI: 10.5281/zenodo.5080993 Released 2021-07-02). UniProtIDs were employed as input type IDs and the total list of Mus musculus genes was used as reference. GO cellular component, molecular function, and cellular component terms with $P<0.05$ with Fisher's Exact were considered significantly enriched.

\section{Statistical Analyses}

Statistical significance was assessed either with parametric statistical test after normality assessment or with nonparametric tests. All analyses were done with GraphPad Prism 9.2.0 (GraphPad Software, Inc.), with $p<0.05$ considered statistically significant.

\section{RESULTS}

\section{TUBB6 Impacts Microtubule Dynamics in Osteoclasts}

To understand how TUBB6 controls actin organization and bone resorption, we generated RAW264.7 cells KO for Tubb6 by CRISPR/Cas9 from which we derived osteoclasts (Figures 1A,B and Supplementary Figure 1A). Similar to what we described in primary osteoclasts treated with Tubb6 siRNAs (Guérit et al., 2020), we found that osteoclasts derived from RAW264.7 Tubb6 KO cells presented several defects when plated on glass: increase of abnormal podosome belts and podosome rings, which are immature podosome belts (Figures 1C,D and Supplementary Figure 1B). Consistently, Tubb6 KO led to osteoclasts with less sealing zones on mineralized substrates and more rings, which are immature sealing zones (Figures 1E,F and Supplementary Figure 1C), as well as reduced resorption activity (Figure 1G). The induction of the expression of osteoclastic markers Src and CtsK was not affected during Tubb6 KO osteoclast differentiation, as assessed by Q-PCR (Supplementary Figures 1D,E). Tartrate-resistant acid protease (TRAP) staining of WT and Tubb6 KO osteoclasts was comparable (Supplementary Figure 1F), as well as Cathepsin K intracellular content and secretion (Supplementary Figure 1G). Finally, the global expression of $\beta$ tubulin (Figure 1A) and the expression of $\beta$ tubulin isotype TUBB5 were also unchanged (Supplementary Figure 1H). Altogether, this shows that RAW264.7 osteoclasts KO for Tubb6 recapitulate the features of primary osteoclasts treated with Tubb6 siRNAs.

To assess the impact of TUBB6 on osteoclast microtubule dynamics, we first performed microtubule regrowth assay consisting in studying microtubule polymerization after cold and nocodazole treatment followed by nocodazole washout. We found that microtubule regrowth was slower in Tubb6 KO osteoclasts (Figures 2A,B). To confirm this, we used Tubb6 siRNAs to lower TUBB6 expression (Guérit et al., 2020). Again, we found that Tubb6 siRNAs reduced the speed

${ }^{3}$ pantherdb.org/ of microtubule regrowth (Supplementary Figure 2). To get additional information regarding this defect, we performed live imaging of osteoclasts expressing the microtubule plus tipbinding protein EB1 fused to GFP (Figure 3A). Using time lapse microscopy and u-track software, we tracked EB1-GFP comets on individual microtubule (Figure 3B and Supplementary Videos 1,2) and measured microtubule dynamic parameters. Thereby, we found a significant decrease of microtubule growth speed in Tubb6 KO osteoclasts from a mean value of $16 \mu \mathrm{m} / \mathrm{min}$ in the wild type (WT) down to $12.9 \mu \mathrm{m} / \mathrm{min}$ in the $\mathrm{KO}$ (Figure 3C and Supplementary Figure 3A). Similar results were found by manual tracking using the MTrack plugin in ImageJ (Supplementary Figures 3B-D). Conversely, we found an increase in microtubule growth time from $18.1 \mathrm{~s}$ in the WT to $20.3 \mathrm{~s}$ in the $\mathrm{KO}$, but no significant change in microtubule growth length (Figure 3C and Supplementary Figure 3A). We also observed that the level of tubulin acetylation, a marker of microtubule stability, was higher in Tubb6 KO osteoclasts (Figures 3D,E), potentially consistent with longer microtubule growth time. Finally, we measured the size of endogenous EB1 staining at microtubule ends, as indicative of the length of the microtubule GTP cap that is known to be proportional to growth speed. We found that the length of EB1 staining was reduced form $1.393 \mu \mathrm{m}$ in WT down to $1.089 \mu \mathrm{m}$ in Tubb6 KO osteoclasts (Figure 3F), consistent with their lower microtubule growth speed (Vemu et al., 2017). The length of the EB1-GFP staining at the end of microtubules was similarly reduced in Tubb6 KO osteoclasts (Supplementary Figures 3E-G).

These results show that TUBB6 has an impact on the parameters of microtubule dynamics, increasing microtubule growth speed while decreasing microtubule lifetime.

\section{TUBB6 Impacts on Actin Dynamics in Osteoclasts}

Actin cytoskeleton and microtubules are intimately linked and Tubb6 KO osteoclasts show abnormal actin cytoskeleton organization. Thus, we further examined the impact of TUBB6 on actin dynamics, by performing live imaging of osteoclasts expressing LifeAct-mCherry to visualize actin cytoskeleton (Figure 4A, Supplementary Figure 4, and Supplementary Videos 3, 4). Major differences in podosome belt dynamics were observed and we thus assessed the stability of the podosome belt by comparing the position of LifeAct-mCherry labeling between time points. In control osteoclasts, the median overlap of LifeActmCherry staining between time 0 and time $1=3$ min was $69.56 \%$, whereas this overlap was significantly reduced to $55.79 \%$ in Tubb6 KO osteoclasts (Figures 4A,B). Similar observations were made at time $2=6.5 \mathrm{~min}$ (Supplementary Figure 4). Finally, we measured the life span of isolated podosomes and found that Tubb6 KO increased to $3.31 \mathrm{~min}$, instead of $2.5 \mathrm{~min}$ in the WT (Figure 4C and Supplementary Videos 5, 6).

This suggests that, on top of microtubules, TUBB6 also impacts on actin dynamics in osteoclasts: TUBB6 tends to reduce podosome life span whereas it increases the stability of the podosome belt. Altogether, the data show that TUBB6, which is required for correct podosome patterning and bone resorption, influences both actin and microtubule dynamics in osteoclasts. 
A

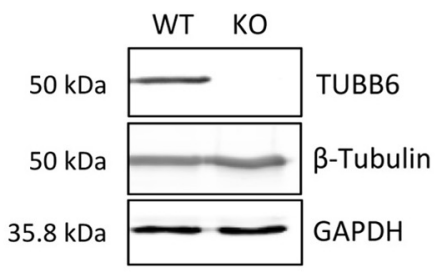

C

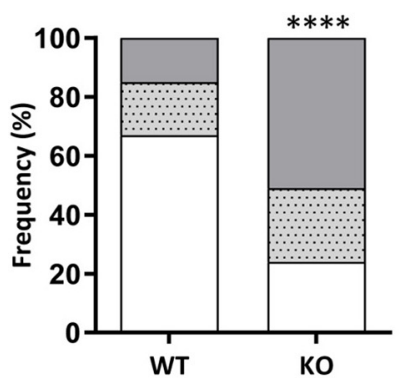

$\square$ Sealing zone

Ring

E

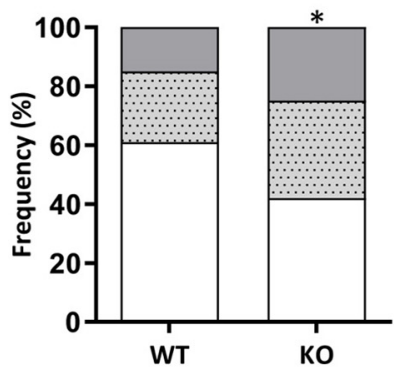

G

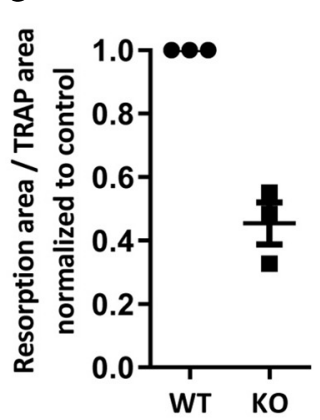

B
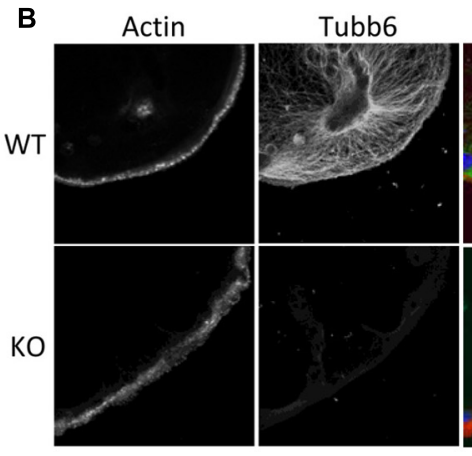

Actin Tubb6

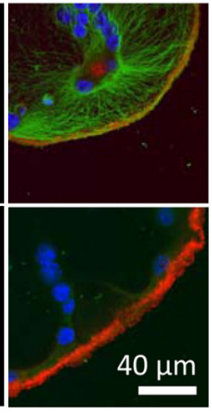

Abnormal

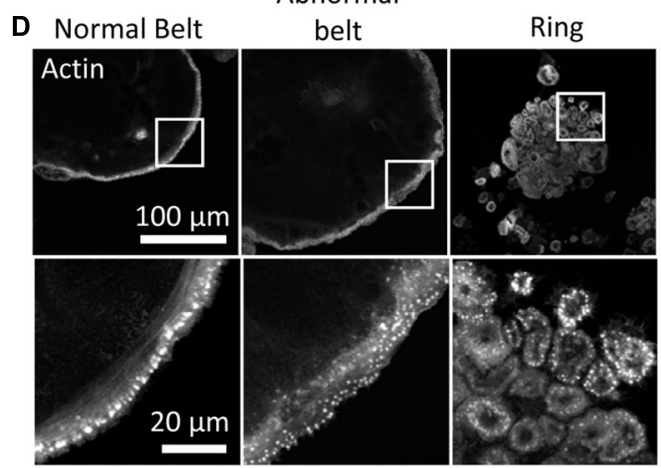

F $\quad \alpha$-tubulin

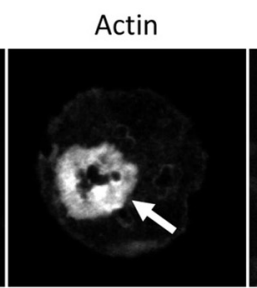

Nuclei

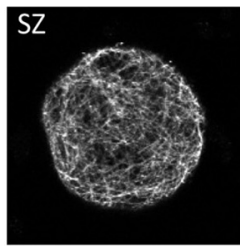

SZ + Ring
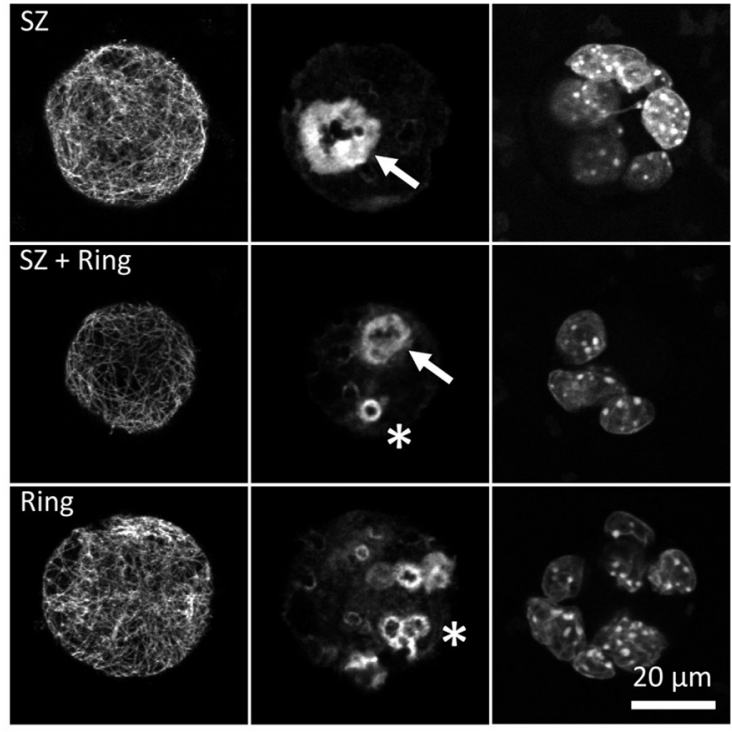

FIGURE 1 | Tubb6 gene knockout alters osteoclast actin cytoskeleton and activity. (A) Representative immunoblot analyses of lysates from wild type (WT) and Tubb6 KO (KO) osteoclasts. GAPDH control shows that total $\beta$-tubulin levels are not affected in osteoclasts that do not express TUBB6. (B) Representative maximum intensity projection (MIP) confocal images of WT and KO osteoclasts seeded on glass and stained for endogenous F-actin and TUBB6. (C) Bar graph showing the frequency of WT and KO osteoclasts seeded on glass and presenting podosome rings, a normal or an abnormal podosome belt, counting more than 200 WT and KO osteoclasts in four independent experiments, $x 2$ contingency test, ${ }^{* * * *} p<0.0001$. (D) Representative MIP images illustrating the different categories of actin organization considered in (C). (E) Bar graph showing the frequency of WT and KO osteoclasts seeded on mineral matrix and presenting either sealing zones (SZ), or rings or both, counting more than $150 \mathrm{WT}$ and KO osteoclasts in three independent experiments, $\chi 2$ contingency test, * $p<0.05$.

(F) Representative MIP images illustrating the different categories of actin organization considered in (E), with SZ and rings respectively indicated with arrows and stars respectively. (G) Graph showing the average and SEM specific resorption activity of KO osteoclasts normalized to the activity of WT osteoclasts in three independent experiments, 4 wells per experiments. Representative image of von Kossa staining of the resorption wells are presented on the right. 

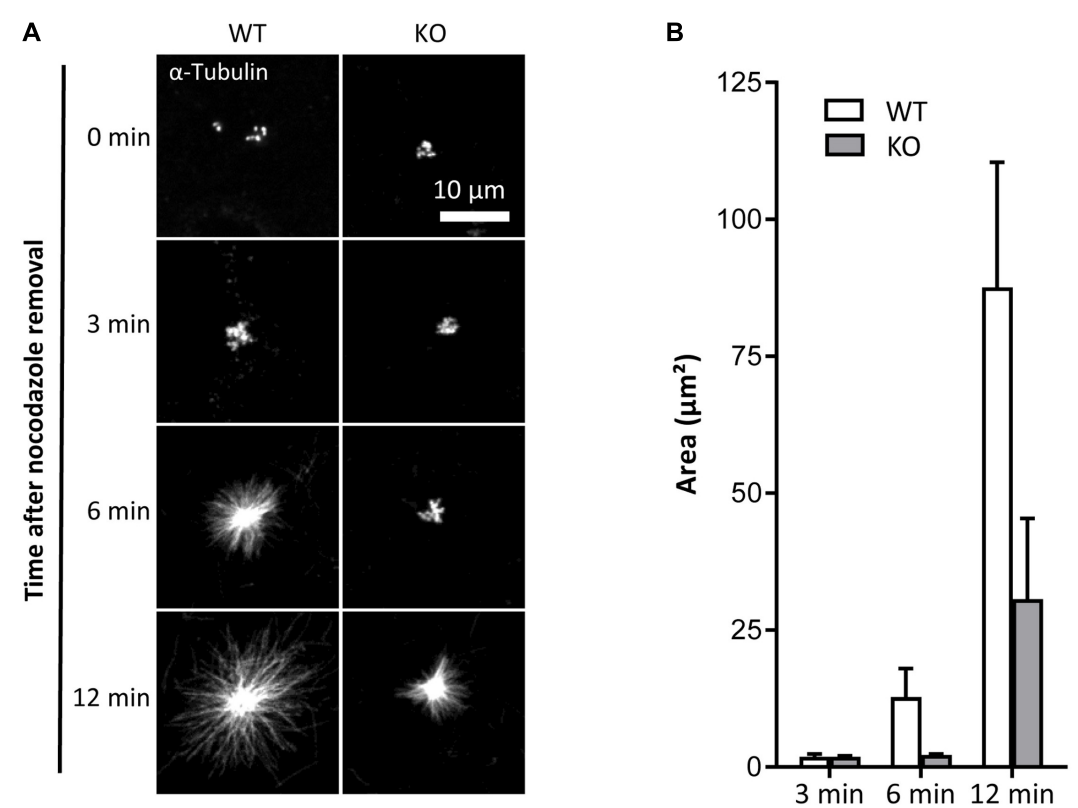

FIGURE 2 | TUBB6 depletion affects MT polymerization. (A) Representative MIP images of WT and KO osteoclasts stained for $\alpha$-tubulin, after release from nocodazole and cold treatment for indicated times. (B) Bar graph showing mean and SEM of $\alpha$-tubulin aster area in WT and KO osteoclasts treated as in (A), 80 WT and $\mathrm{KO}$ asters in four independent experiments.

\section{TUBB6 Moderately Affects Protein Binding to Osteoclast Microtubules}

To understand the impact of TUBB6 on osteoclast cytoskeleton, we examined whether Tubb6 KO could affect protein binding to osteoclast microtubules. For this, we thought to prepare osteoclast fractions enriched in microtubule-associated proteins (Figure 5A). Osteoclasts lysates were prepared at room temperature, in a paclitaxel-free microtubule-preserving hypotonic buffer. Lysates were centrifuged to separate the supernatant (S1) from the pellet. The pellet was then washed with the same buffer supplemented with $350 \mathrm{mM} \mathrm{NaCl}$, GTP and paclitaxel, to detach microtubule-bound proteins (S2) from microtubules while preserving the latter, which were finally pelleted (P) (Figure 5A). Immunoblot analysis of the different fractions showed the expected marker distribution: total tubulin equally distributed in $\mathrm{S} 1$ and $\mathrm{P}$ whereas acetylated tubulin fell predominantly in $\mathrm{P}$, which contains microtubules; the microtubule-associated protein CLASP1 was found in S2 whereas vinculin was predominantly in S1 (Figure 5B). Of note, all fractions contained similar amounts of actin, indicative of partial F-actin depolymerization in $350 \mathrm{mM} \mathrm{NaCl}$ (Figure 5B). Finally, histone $\mathrm{H} 3$ was found in the pellet, in agreement with the presence of nuclei in this fraction; small amounts of histone $\mathrm{H} 3$ were also found in S2, likely due to nuclear protein leaking out of the nucleus upon high salt incubation after hypotonic lysis of osteoclasts (Figure 5B).

We performed label-free proteomics to compare protein content in the S2 fractions of WT and Tubb6 KO osteoclasts, in four independent experiments. We identified 2799 protein groups, considering two peptides for adequate protein identification, among which 2778 corresponded unambiguously to a single Uniprot identifier and to a unique protein (Supplementary Table 1). To examine whether S2 fraction was enriched in cytoskeleton regulatory proteins, we used Panther 16.0 to perform gene ontology analyses of the 2773 proteins (5 Uniprot identifiers could not be mapped in Panther), as compared to the total mouse genome (Supplementary Table 2). Using actin, tubulin, microtubule, and cytoskeleton as keywords, we found that all Gene Ontology (GO) terms associated with the cytoskeleton in molecular functions, cellular components, and biological process showed a significant enrichment in the S2 fraction (Supplementary Table 2). Cytoskeleton-related proteins associated with these GO terms encompassed 601 of the 2773 proteins we analyzed in Panther (Supplementary Table 3). Thus, S2 contains a great proportion of proteins related to microtubules and actin cytoskeleton.

Applying a twofold change cutoff with a $p$-value below $10^{-2}$, we found only seven proteins showing significant changes in the S2 of Tubb6 KO osteoclasts as compared to WT (Figure 5C and Table 1). Among these, only two were part of the 601 proteins associated with cytoskeleton-related GO terms: the podosomeassociated protein LSP1 (lymphocyte specific 1) and the Rhofamily GTPase regulating protein ARHGAP10 (also known as GRAF2 or PS-GAP) (Figure 5C and Table 1).

These results show that TUBB6 has little effect on the content of the osteoclast S2 fraction, which is enriched in microtubuleassociated protein. In particular, among the 601 proteins present in S2 and associated with a cytoskeleton-related GO term, only LSP1 and ARHGAP10 showed significant changes. This suggests that TUBB6 can affect the interaction of LSP1 and ARHGAP10 with F-actin and/or microtubules. 

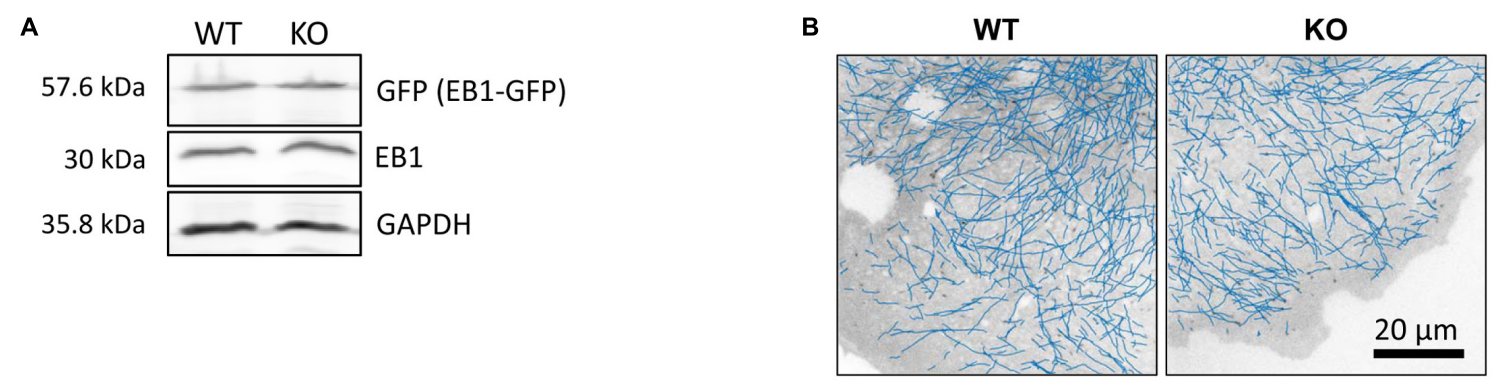

C
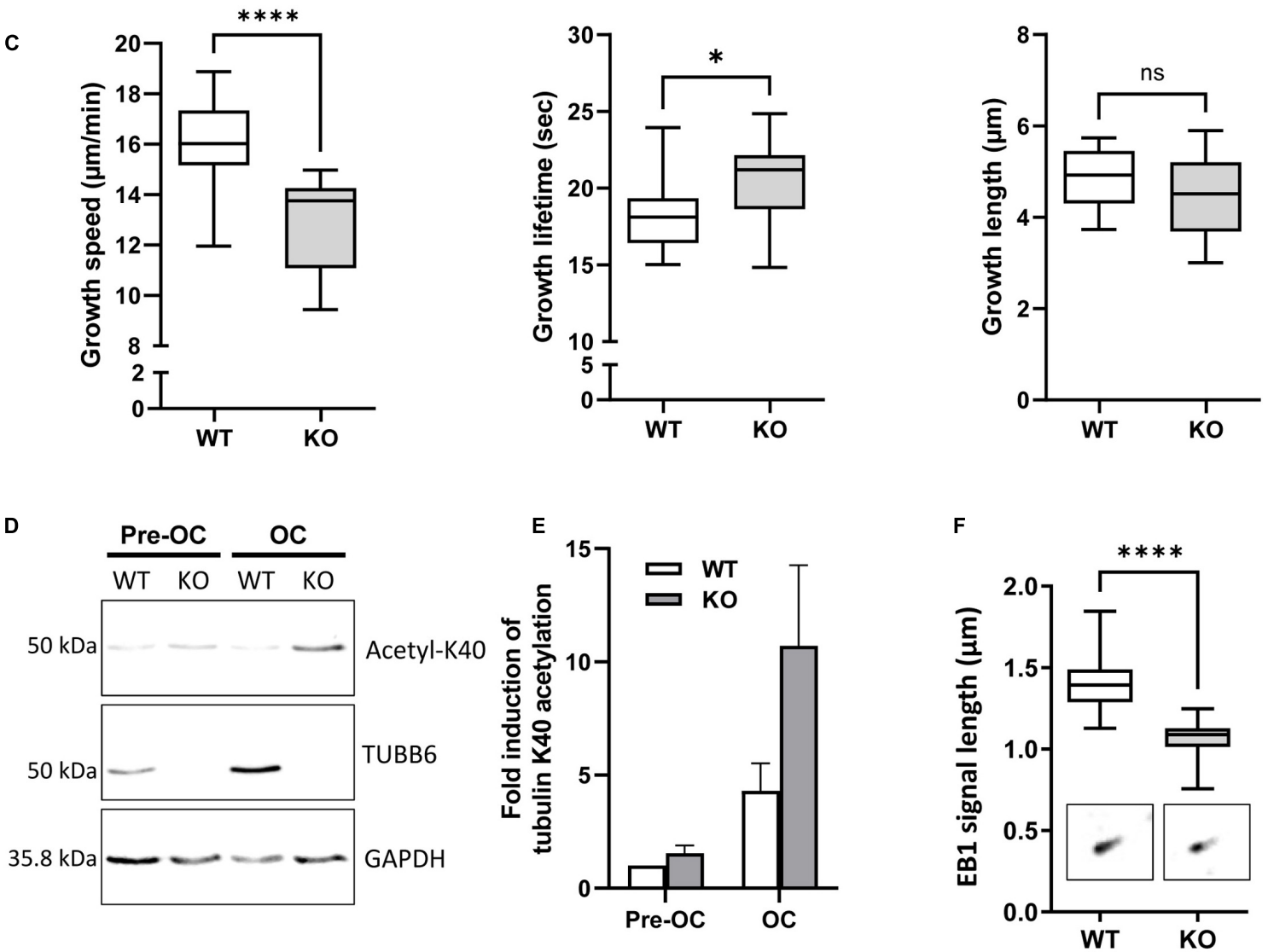

FIGURE 3 | TUBB6 regulates MT dynamics. (A) Representative immunoblot analysis of protein lysates from WT and KO osteoclasts expressing EB1-GFP (GFP); note that the expression levels of EB1-GFP and endogenous EB1 are similar in WT and KO osteoclasts. (B) Blue lines show representative EB1-GFP comet tracking using u-track software in WT and KO osteoclasts from confocal images over a period of 2 min with $1 \mathrm{~s}$ increment between frames (see section "Materials and Methods" for detailed settings). (C) Minimum to maximum boxplots with median and interquartile range (25-75\%) showing the distribution of growth speed (left), lifetime (middle) and length (right) of EB1-GFP tracked comets per single WT and KO osteoclast, 18 WT and 14 KO osteoclasts from 3 independent experiments; Mann Whitney test: ${ }^{* * * *} p<0.0001$ and ${ }^{*} p<0.05$. For details see table in Supplementary Figure 3A. (D) Representative immunoblot analysis of protein lysates from WT or KO RAW264.7 cells (Pre-OC) and derived osteoclasts (OC), showing TUBB6, acetylated K40 $\alpha$-tubulin (acetyl-K40), and GAPDH expression. (E) Bar graph shows mean and SEM levels of acetylated K40 $\alpha$-tubulin in WT and KO pre-OC and OC, normalized to 1 in WT pre-osteoclasts, from four independent experiments. (F) Minimum to maximum boxplot showing endogenous EB1 cap length in WT and KO osteoclasts, measuring 25 comets in each osteoclast to determine the average cap length per osteoclast, in 27 WT and $27 \mathrm{KO}$ osteoclasts from three different experiments; Mann-Whitney test: ${ }^{* * * *} p<0.0001$.

\section{ARHGAP10 Associates With Both Actin and Microtubules in Osteoclasts}

ARHGAP10 was shown to be a negative regulator of the activity of the GTPases CDC42, in a PYK2-kinase dependent fashion (Ren et al., 2001; Koeppel et al., 2004); ARHGAP10 can also regulate negatively the activity of GTPase RHOA (Ren et al., 2001). It was reported in RAW264.7-derived osteoclasts that Arhgap10 siRNAs led to smaller sealing zones, without affecting the proportion of osteoclasts with a sealing zone (Steenblock et al., 2014). Interestingly, we also observed smaller sealing zones upon Tubb6 KO (Figure 1 and Supplementary Figure 1), as well as with Tubb6 siRNAs in primary mouse and human osteoclasts (Guérit et al., 2020). We found that ARHGAP10 expression was strongly induced during both WT 


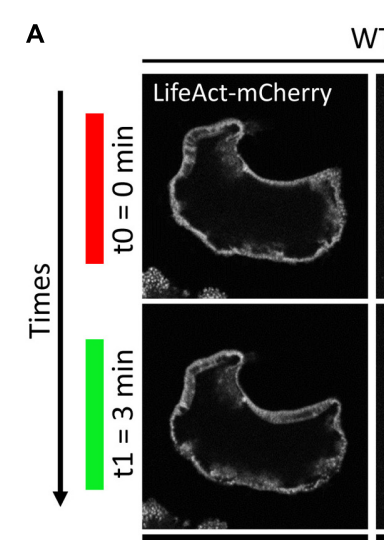

WT
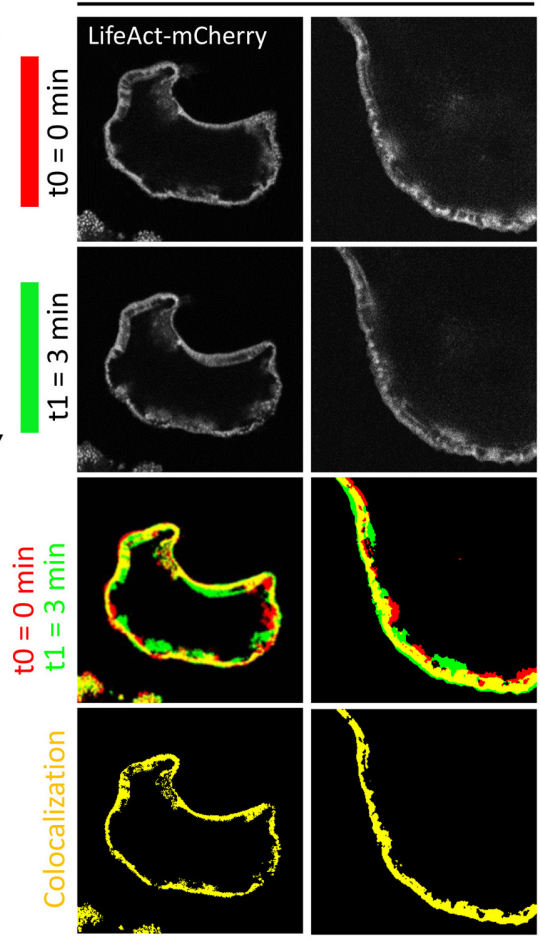

B

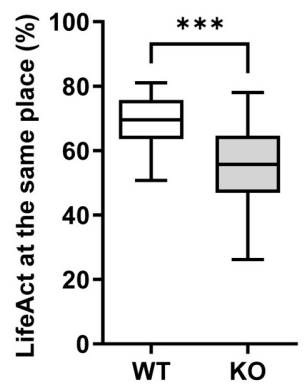

KO
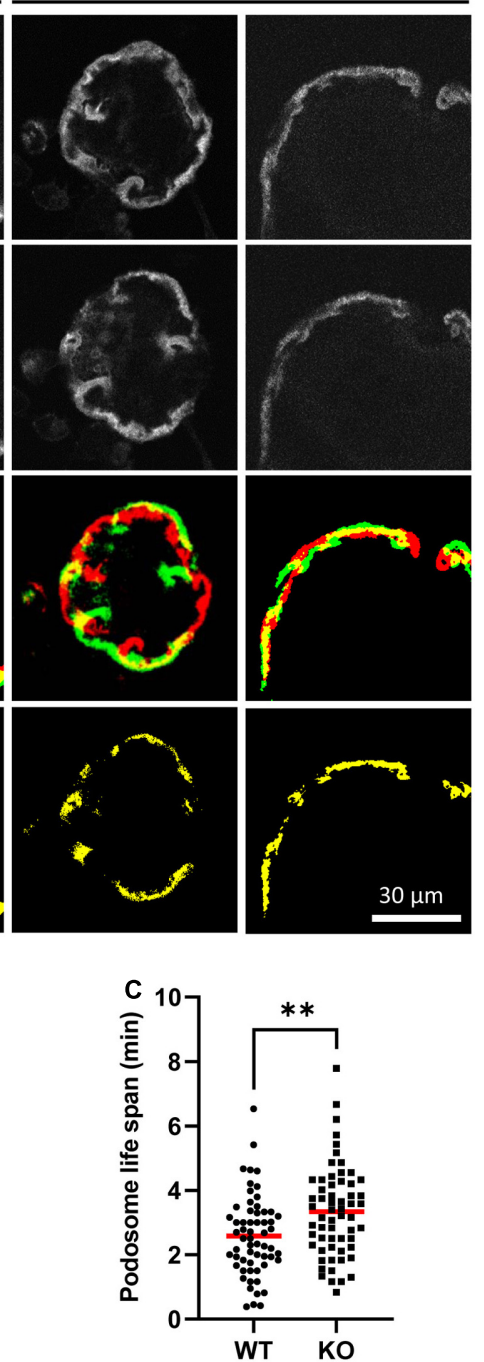

FIGURE 4 | TUBB6 depletion affects podosome belt dynamics. (A) Representative live confocal images of osteoclasts derived from WT or Tubb6 KO osteoclasts expressing LifeAct-mCherry and sitting on glass, showing the localization of LifeAct-mCherry signal at t0 = 0 min (red) and t $1=3$ min (green), with the overlapping areas in yellow. (B) Minimum to maximum boxplot showing the percentage of LifeAct-mCherry signal at t0 that persists at the same position at $\mathrm{t3}$ in WT or Tubb6 KO osteoclasts; in a total of 22 WT and $21 \mathrm{KO}$ osteoclasts from 4 different experiments. Mann-Whitney test: *** $p<0.0002$. See also Supplementary Figure 4 and Supplementary Videos 3, 4. (C) Dot plot showing the life span with mean of individual podosomes in WT or Tubb6 KO osteoclasts. Total of 60 podosomes in six different wild type and $\mathrm{KO}$ osteoclasts, from three independent experiments. Student's $t$-test: ${ }^{* *} p<0.002$. See also Supplementary Videos $\mathbf{5}, \mathbf{6}$.

and Tubb6 KO osteoclasts (Figure 5D). Endogenous ARHGAP10 colocalized with the podosome belt in RAW264.7-derived and primary osteoclasts, as well as with the sealing zone of osteoclasts plated on ACC (Figures 6A,B). Interestingly, we also observed endogenous ARHGAP10 colocalization with osteoclast microtubules (Figures 6C,D). To confirm that ARHGAP10 can interact with microtubules, we overexpressed ARHGAP10 fused to a HA-GST tag in HEK293T cells, which do not express endogenous ARHGAP10 (Koeppel et al., 2004), or HA-GST as a control. After lysis in a buffer compatible with microtubule polymerization and clarification by high-speed centrifugation, lysates were either incubated at $4^{\circ} \mathrm{C}$ or $37^{\circ} \mathrm{C}$ in the presence of GTP and paclitaxel to induce microtubule polymerization.
Finally, the fraction of HA-GST fused proteins co-sedimenting with microtubules were analyzed by immunoblot after a final high-speed centrifugation. Tubulin was found only in the $37^{\circ} \mathrm{C}$ pellet, indicating that no microtubule polymerized at $4^{\circ} \mathrm{C}$. As expected, HA-GST was only retrieved in supernatants regardless the temperatures (Figure 6E). Interestingly, at $37^{\circ} \mathrm{C}, \mathrm{HA}-\mathrm{GST}$ ARHGAP10 almost exclusively co-sedimented with microtubules whereas it was retained in the supernatant when the experiment was performed at $4^{\circ} \mathrm{C}$ to prevent microtubule polymerization (Figure 6F). Of note, very little actin was retrieved in the osteoclast lysate and no actin could be detected in the pellets that contained ARHGAP10 (Figure 6F). These data show that ARHGAP10 is indeed a microtubule-associated protein. 
A

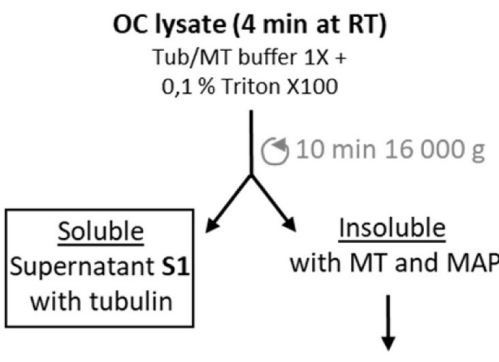

MAP elution with $\mathrm{NaCl}$

Tub/MT buffer $1 \mathrm{X}+$ $20 \mu \mathrm{M}$ taxol $+0,1 \mu \mathrm{M} \mathrm{GTP}+350 \mathrm{mM} \mathrm{NaCl}$

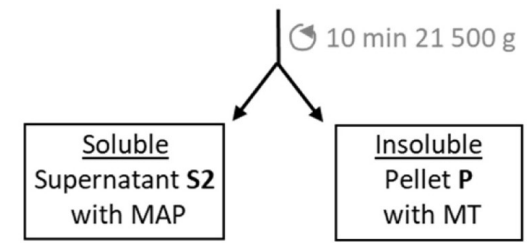

Proteomic analysis

\section{C}

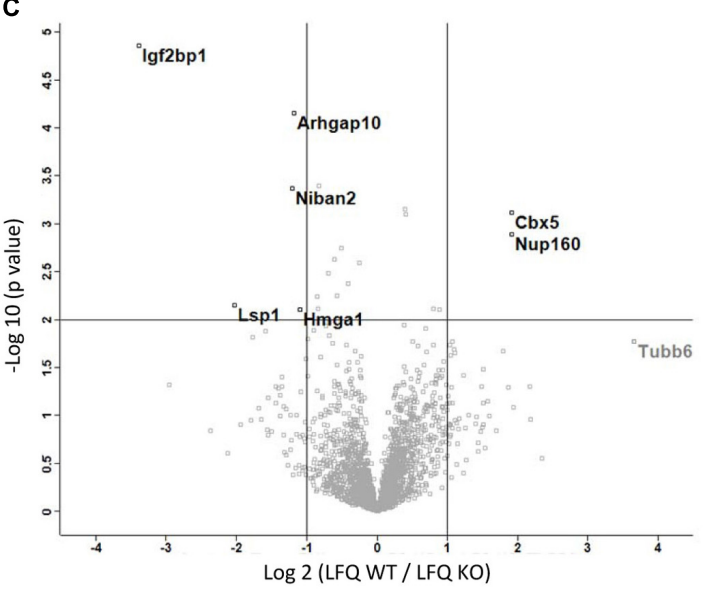

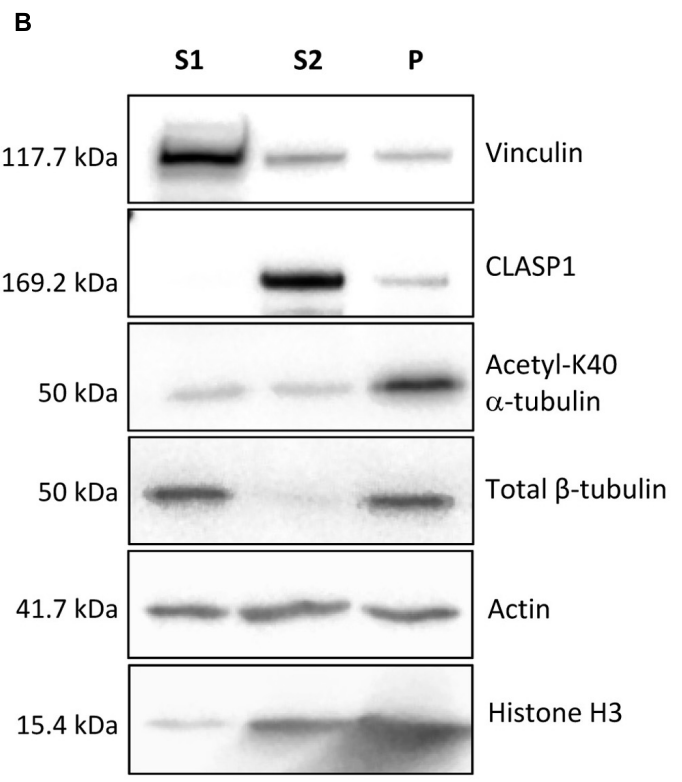

D

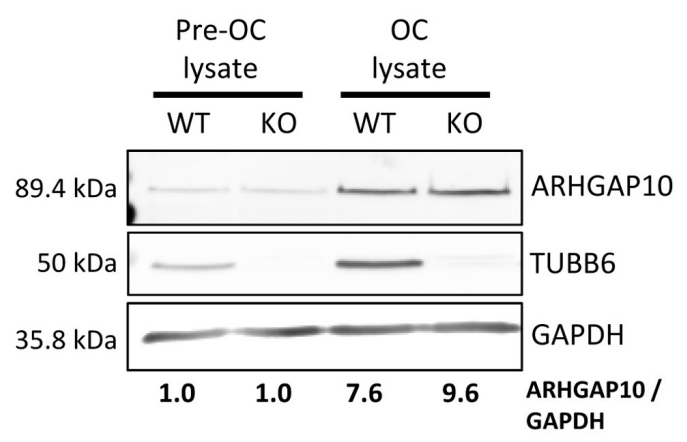

FIGURE 5 | Characterization of the microtubule-associated protein fraction of WT and Tubb6 KO osteoclasts. (A) Schematic representation of the osteoclast fractionation strategy in microtubule-stabilizing Tub/MT buffer to obtain soluble proteins (S1), a fraction enriched in microtubule-binding protein (S2) and an insoluble fraction containing microtubules (P). (B) Representative immunoblot analysis of selected protein distribution between the different fractions: Vinculin, CLASP1, acetylated K40 $\alpha$-tubulin, $\beta$-tubulin, actin, and Histone H3. (C) Volcano plot of Label Free Quantitation (LFQ) results from four independent mass spectrometry experiments, showing proteins that are significantly more abundant in $\mathrm{S} 2$ from $\mathrm{KO}$ (top left) and WT (top right) osteoclast, with vertical lines indicating twofold change cut off and horizontal line $p=0.01$ cut off, with $x$ axis in $\log _{2}\left(\mathrm{LFQ}\right.$ WT/LFQ KO) and $y$ axis in $-\log _{10}(p$-value). (D) Representative immunoblot analysis of ARHGAP10, TUBB6 and GAPDH expressions in WT or Tubb6 KO RAW264.7 cells (Pre-OC) and in the osteoclasts (OC) derived from these cells.

Altogether, our data suggest that TUBB6 can control the amount of ARHGAP10 associated with microtubules in osteoclasts. This may contribute to control the size of sealing zones, which was shown to involve both TUBB6 (Guérit et al., 2020) and ARHGAP10 (Steenblock et al., 2014).

\section{DISCUSSION}

The cytoskeleton plays a key role in bone resorption by osteoclasts. F-actin organizes as a belt of podosomes required for adhesion and as backbone of the ruffled border, which is the actual bone resorption apparatus. Microtubules are also instrumental for podosome organization in osteoclasts and bone resorption, but the coordination mechanisms between the actin and tubulin cytoskeletons remain poorly characterized.

We identified previously TUBB6 as a $\beta$-tubulin isotype essential in osteoclasts for podosome belt integrity and for resorption (Guérit et al., 2020). To study the implications of TUBB6 in cytoskeleton dynamic parameters, we developed a model of osteoclasts KO for Tubb6 using the RAW264.7 pre-osteoclast cell line, which recapitulates the phenotype we 

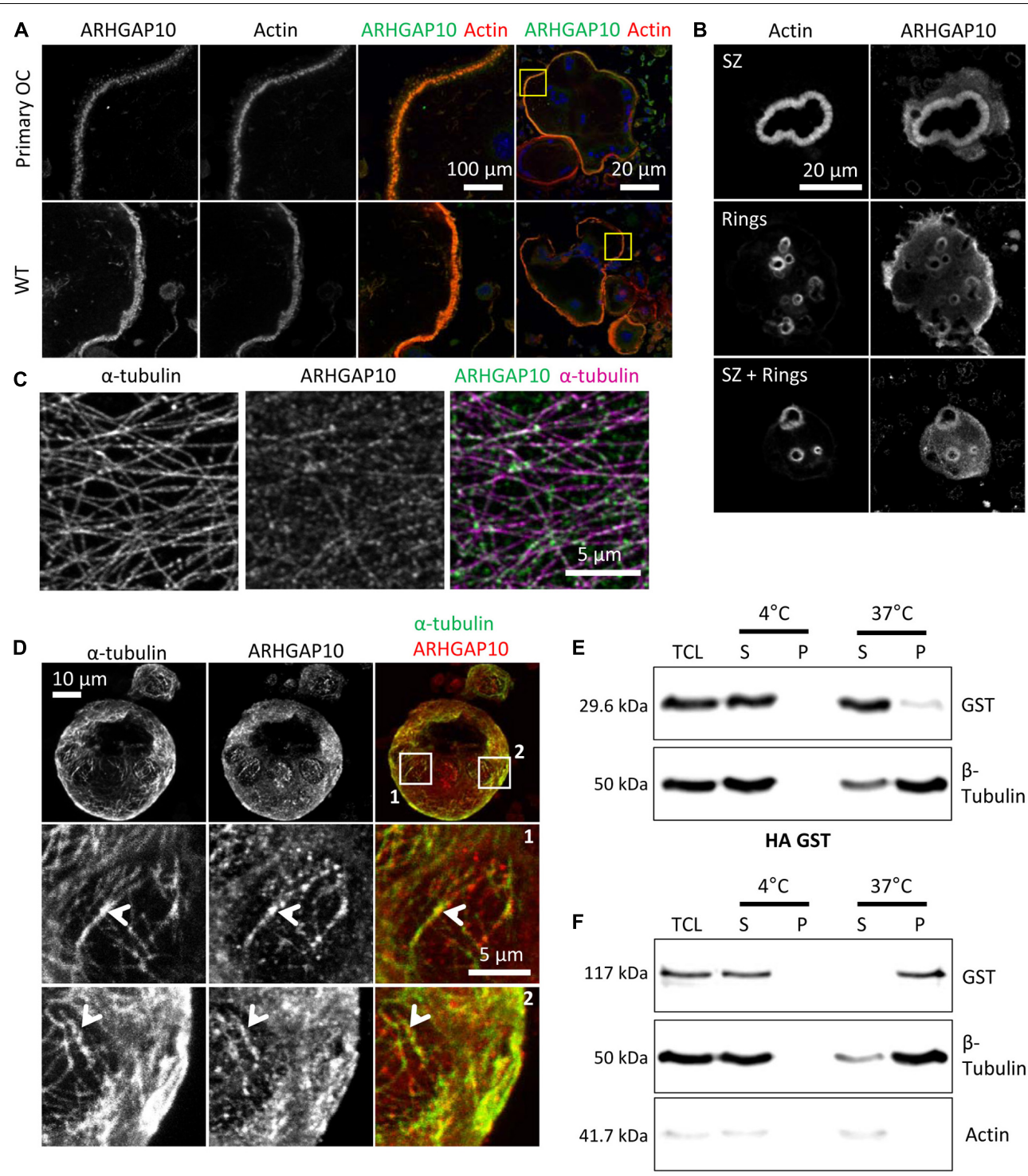

HA GST-ARHGAP10

FIGURE 6 | ARHGAP10 is associated with both actin and microtubules. (A) Representative MIP images of RAW264.7 derived or primary (primary OC) osteoclasts seeded on glass and stained for ARHGAP10, actin, and DNA. Left images show enlargement of boxed areas in the images on the right. (B) Representative MIP images showing ARHGAP10 and actin localization in WT and Tubb6 KO osteoclasts sitting on ACC. (C) Representative single plan confocal image of a WT osteoclast stained for ARHGAP10 (green) and $\alpha$-tubulin (pink). (D) Representative MIP images of a WT osteoclast sitting on ACC and stained for ARHGAP10 and $\alpha$-tubulin. Boxed areas in top images are enlarged below, to show ARHGAP10 colocalization with microtubules (arrowheads). (E,F) Immunoblot analysis of Iysates from HEK293T cells expressing HA-GST (E) or HA-GST-ARHGAP10 (F), and subjected to microtubule pelleting assays at $4^{\circ} \mathrm{C}$ or $37^{\circ} \mathrm{C}$. ARHGAP10 co-sediments at $37^{\circ} \mathrm{C}$ with microtubules in the pellet, which contains $\beta$ tubulin but is devoid of actin.

reported recently for primary osteoclasts treated with Tubb6 siRNAs (Guérit et al., 2020). On top of the expected abnormal actin organization and reduced resorption activity, we found that the lack of TUBB6 also effected microtubule dynamics in osteoclasts: the speed of microtubule repolymerization after nocodazole treatment was reduced as well as individual microtubule growth speed, whereas their lifetime was increased, accompanied by an increase in tubulin acetylation. This suggests that TUBB6 has a microtubule-destabilizing effect in osteoclasts. Interestingly, overexpression of TUBB6 was reported previously to disrupt microtubule organization in various cycling cell types (Bhattacharya and Cabral, 2004) and to induce paclitaxel resistance, with serine 239 being essential for TUBB6 microtubule-destabilizing activity (Bhattacharya and Cabral, 2009). Serine 239 is located within the core of $\beta$-tubulin and it is conserved in TUBB1 and TUBB3. Interestingly, similarly to TUBB6, high expression of TUBB3 is suspected to participate in paclitaxel resistance (Kato et al., 2018). Furthermore, TUBB3 has been shown to significantly decrease microtubule growth rates (Pamula et al., 2016; Vemu et al., 2016), similar to what we 
TABLE 1 | Protein changes in fractions S2.

\begin{tabular}{|c|c|c|c|c|c|c|}
\hline Uniprot ID & Protein name & Gene name & Peptide counts & Coverage $\%$ & LFQ fold change KO/WT & $P$-value \\
\hline \multicolumn{7}{|c|}{ More abundant in Tubb6 KO osteoclast S2 } \\
\hline O88477 & Insulin-like growth factor 2 mRNA-binding protein 1 & lgf2bp1 & 9 & 20.1 & 10.44 & $1.39 \mathrm{E}-05$ \\
\hline P19973 & Lymphocyte-specific protein 1 & Lsp1 & 5 & 27.9 & 4.06 & 7.16E-03 \\
\hline Q8R1F1 & Protein Niban 2 & Niban2 & 23 & 42.3 & 2.30 & 4.27E-04 \\
\hline Q6Y5D8 & Rho GTPase-activating protein 10 & Arhgap10 & 24 & 36.1 & 2.27 & 7.00E-05 \\
\hline P17095 & High mobility group protein HMG-I/HMG-Y & Hmga1 & 7 & 48.6 & 2.14 & 7.90E-03 \\
\hline \multicolumn{7}{|c|}{ More abundant in WT osteoclast S2 } \\
\hline Q9Z0W3 & Nuclear pore complex protein Nup160 & Nup160 & 6 & 6.9 & 0.26 & 1.30E-03 \\
\hline Q61686 & Chromobox protein homolog 5 & Cbx5 & 6 & 40.3 & 0.26 & 7.61E-04 \\
\hline
\end{tabular}

found here for TUBB6. Furthermore, this intrinsic property on microtubule dynamics does not require residues located within the carboxy-terminal tail of TUBB3 leaving the possibility of serine 239 involvement (Pamula et al., 2016). Based on these data, it is very likely that TUBB6 intrinsically alters the dynamics of microtubules, similar to TUBB3.

We noticed an increase of K40-acetylation of $\alpha$-tubulin upon $\mathrm{KO}$ of Tubb6 in osteoclasts, as also observed in $\mathrm{CHO}$ cells expressing Tubb6 shRNAs (Bhattacharya et al., 2011). K40acetylation of $\alpha$-tubulin is a well described marker of stable MT (Janke and Magiera, 2020). The increase of K40-acetylation we observed in Tubb6 KO osteoclasts may thus be a consequence of the increase of MT lifetime that we measured. K40-acetylation has been shown to play a crucial role in podosome belt establishment and/or stability in osteoclasts (Destaing et al., 2005). Lower K40-acetylation levels were also associated with various genetic defects leading to the alteration of podosome patterning in osteoclasts, such as the KO of Pyk2, myosin IXB, or cofilin 1 (Gil-Henn et al., 2007; McMichael et al., 2014; Zalli et al., 2016). In the case of Tubb6 gene disruption, we found conversely an increase of K40-acetylation associated with an abnormal organization of podosomes. Microtubule acetylation was shown to enhance their flexibility (Portran et al., 2017) and actually microtubules are heavily buckled in the podosome belt area of primary osteoclasts treated with Tubb6 siRNAs as compared to control (Guérit et al., 2020). Thus, an optimal level of microtubule acetylation appears essential for osteoclast function, potentially to provide microtubules with appropriate flexibility and mechanical resistance (Portran et al., 2017; Xu et al., 2017). Such optimal balance is known for other PTMs in general and for microtubule PTMs in particular. For instance, increasing and decreasing the level of microtubule glutamylation equally impair ciliary function in the nematode (O'Hagan et al., 2017). K40acetylation could also participate in the changes in podosome dynamics we observed in Tubb6 KO osteoclasts. Indeed, dynamic microtubules were shown to regulate podosome fate in macrophages: podosome contact by plus-end MT mostly induces their dissolution or fission (Kopp et al., 2006) and increasing K40acetylation of microtubules diminishes microtubule plus ends targeting of podosomes (Bhuwania et al., 2014). In Tubb6 KO osteoclasts, we observe increased podosome life span correlated with increased microtubule acetylation, which may result from a reduction in contacts between microtubules and podosomes.
Apart from directly impacting on the dynamics of microtubules, tubulin isotypes influence the recruitment of proteins on microtubules, which can also modulate their dynamics as well as cargo transport (Janke and Magiera, 2020). Our proteomic analyses of osteoclast S2 fractions, enriched in microtubule-associated proteins, did not reveal major changes whether osteoclasts expressed TUBB6 or not. Still, we found two cytoskeleton-related proteins: ARHAP10 and LSP1, which were increased in $\mathrm{S} 2$ in the absence of TUBB6. Similar to what was reported with Arhgap10 siRNAs (Steenblock et al., 2014), we found that the osteoclast $\mathrm{KO}$ of Tubb6 have small sealing zones that often remain immature. ARHGAP10 is a GAP that negatively regulates the activity of CDC42 (Ren et al., 2001). It was shown in osteoclasts to participate in a complex with the CDC42 exchange factor FGD6, all three proteins localizing at the sealing zone (Steenblock et al., 2014). Our data show that ARHGAP10 also colocalizes with osteoclast microtubules and that ARHGAP10 associates very efficiently with microtubules in an actin-independent fashion, suggesting that ARHGAP10 can associate with both microtubules and actin cytoskeleton in osteoclasts. CDC42 is essential for the correct dynamics of actin rings in osteoclasts and for bone resorption in vitro and in vivo in the mouse; conversely, excessive activity of CDC42 causes osteoclast hyperactivity and osteoporosis (Ito et al., 2010). The global level of CDC42 activity was not affected in osteoclasts $\mathrm{KO}$ for Tubb6 as compared to WT (data not shown), suggesting that by controlling ARHGAP10 association with the cytoskeleton, TUBB6 could contribute to the fine tuning of local CDC42 activity to ensure an optimal level of resorption. LSP1 is an actin-regulating protein, which was recently shown to associate with the cap of podosomes in macrophages (Cervero et al., 2018). LSP1 regulates macrophage podosome properties: the levels of LSP1 control the frequency and amplitude of podosome oscillations, and interfering with LSP1 expression reduces podosome life span and the protruding forces they exert on the extracellular matrix (Cervero et al., 2018). Moreover, lack of LSP1 was recently shown to affect the microtubule network in macrophages (Schäringer et al., 2021). Here we found that in the absence of TUBB6, podosome have longer life-span and we found increased amounts of LSP1 in the S2 fraction of Tubb6 KO osteoclasts, suggesting that LSP1 may participate in the control of actin-dynamics in osteoclasts in a TUBB6-dependent 
fashion. The function of LSP1 in osteoclasts would deserve further investigations.

To conclude, our data highlight the crucial role of microtubule dynamics in podosome belt integrity in general and the involvement of TUBB6 in particular. We provide new insights into the control of microtubule dynamics in osteoclasts and position $\beta$-tubulin isotype TUBB6 as a key regulator of the crosstalk between actin and microtubules in osteoclast. Our results uncover novel regulatory mechanisms of osteoclast cytoskeleton and contribute to the better knowledge of the molecular processes controlling bone resorption. Our data could be valuable also in the context of osteolytic bone diseases and pave the way for novel solutions against osteoporosis by targeting osteoclast cytoskeleton.

\section{DATA AVAILABILITY STATEMENT}

Proteomic data from Max Quant treated with Perseus are in Supplementary Table 1.

\section{AUTHOR CONTRIBUTIONS}

$\mathrm{AB}$ and $\mathrm{GB}$ : conceptualization and supervision. $\mathrm{AB}, \mathrm{GB}, \mathrm{JM}$, AM, DG, JC, and SU: methodology. GB, JM, AM, DG, JC, and SU: validation. AB, GB, JM, AM, DG, and SU: formal analyses. GB, JM, AM, DG, and SU: investigation. AB, GB, JM, and SU: writing - original draft. $\mathrm{AB}$ : project administration and funding acquisition. All authors contributed to the article and approved the submitted version.

\section{FUNDING}

This study was supported by the Centre National de la Recherche Scientifique (CNRS) and by the Universiteì de Montpellier, and by grants from the Fondation pour la Recherche Meidicale (DEQ20160334933 to AB), the Fondation ARC pour la Recherche sur le Cancer (PJA 20191209321 to AB), and the Ligue Contre le Cancer Comité de l'Hérault (Grant LNCC HERAULT LS 1995452020 to $\mathrm{AB})$.

\section{ACKNOWLEDGMENTS}

We acknowledge the imaging facility Montpellier Ressources Imagerie (MRI), a member of the national infrastructure France-BioImaging supported by the French National Research Agency (ANR-10-INBS-04, 'Investments for the future'). Mass spectrometry experiments were carried out using the facilities of the Montpellier Proteomics Platform (PPM, BioCampus Montpellier). We are very grateful to Ariane Abrieu, Leslie Bancel-Vallée, Daniel Bouvard, Juliette van Dijk, Nathalie Morin, Didier Portran, and Amélie Viel (CRBM Montpellier, France) to for technical assistance, helpful advice and discussions. We also thank Daisuke Mori (Nagoya University, Japan) for the generous gift of ARHGAP10 expression vector.

\section{SUPPLEMENTARY MATERIAL}

The Supplementary Material for this article can be found online at: https://www.frontiersin.org/articles/10.3389/fcell.2021. 778887/full\#supplementary-material

Supplementary Figure 1 | (A) Representative immunoblot showing TUBB6 and GAPDH expression in WT and Tubb6 KO RAW264.7 clones. Note in overexposed panel in the middle that clones 2 and 4 retain traces of TUBB6. (B) Bar graph showing the frequency of WT and KO osteoclasts seeded on glass and presenting podosome rings, normal or abnormal podosome belt, counting a total of 200 WT and $\mathrm{KO}$ osteoclasts in two independent experiments. (C) Bar graph showing the frequency of $\mathrm{WT}$ and $\mathrm{KO}$ osteoclasts seeded on $\mathrm{ACC}$ and presenting sealing zone and/or rings counting over 50 osteoclasts per clone. (D,E) Bar graphs showing the level of osteoclast characteristic Cathepsin K (D) and Src (E) mRNAs relative to Gapdh as determined by Q-PCR in WT or Tubb6 KO RAW264.7 cells (Pre-OC) or osteoclasts (OC). (F) Representative images showing TRAP staining in WT and Tubb6 KO osteoclasts. (G) Representative immunoblot blot showing intracellular content of alpha-tubulin, Cathepsin $\mathrm{K}$ proenzyme, and its mature form in total cell lysates of WT and Tubb6 KO osteoclasts, and the secretion of mature Cathepsin $\mathrm{K}$ in the culture medium of the same cells. (H) Representative immunoblot blot showing TUBB5, TUBB6 and GAPDH expression in primary bone marrow macrophages (Pre-OC) and osteoclasts (OC) and in WT or Tubb6 KO RAW264.7 cells (pre-OC) or osteoclasts (OC).

Supplementary Figure $\mathbf{2}$ | Bar graph showing mean and SEM $\alpha$-tubulin aster area after nocodazole washout in osteoclasts transfected with luciferase control siRNA or Tubb6 siRNAs si1077 and si1373, measuring at least 20 asters per group were measured in four independent experiments.

Supplementary Figure 3 | (A) Table showing the details of microtubule dynamics parameters from u-track analysis (B) Minimum to maximum boxplot showing microtubule growth speed determined in ImageJ 1.53c with MTrackJ in 15 WT and Tubb6 KO osteoclasts expressing EB1-GFP from three independent experiments and measuring 10-11 comets per osteoclasts; Mann-Whitney test: ${ }^{* * *} p<0.0002$. (C,D) Representative EB1-GFP comets tracks in WT (C) and Tubb6 KO (D) analyzed in (B). (E) Minimum to maximum boxplot showing the length of the EB1-GFP signal in 18 WT and Tubb6 KO osteoclasts per condition from three independent experiments and measuring 25 comets in each osteoclast to determine the average comet length per osteoclast; Mann-Whitney test: $* * * * p<0.0001$. $(\mathbf{F}, \mathbf{G})$ Representative images of the EB1-GFP signal at the tip of microtubules in WT (F) and KO (G) osteoclasts analyzed in (E).

Supplementary Figure 4 | (A) Representative live confocal images of osteoclasts derived from WT or Tubb6 KO osteoclasts expressing LifeAct-mCherry and sitting on glass, showing the localization of LifeAct-mCherry signal at to $=0 \mathrm{~min}$ (red) and t2 $=6.5$ min (green), with the overlapping areas in yellow. (B) Minimum to maximum boxplot showing the percentage of LifeAct-mCherry signal at to that persists at the same position at t2 in WT or Tubb6 KO osteoclasts; in a total of 22 WT and $21 \mathrm{KO}$ osteoclasts from four different experiments. Mann-Whitney test: $* * p<0.01$.

Supplementary Table 1 | Proteomic data from Max Quant treated with Perseus.

Supplementary Table 2 | Gene Onthology analysis of the proteins in the S2 fraction.

Supplementary Table $\mathbf{3}$ | List of the 601 proteins of the S2 fraction associated with a cytoskeleton-related Gene Ontology term.

Supplementary Videos 1, 2 | Detection and tracking of EB1-GFP expressing osteoclasts WT (video 1) or KO (video 2) using u-track software and settings described in Materials and Methods. Time is indicated as min:sec and frame is $68 \mu \mathrm{m}$.

Supplementary Videos 3, 4 | Live imaging of LifeAct-mCherry expressing WT (video 3) or KO (video 4) osteoclasts. Time is indicated as min:sec and frame is $90.5 \mu \mathrm{m}$.

Supplementary Videos 5, 6 | Live imaging of LifeAct-mCherry expressing WT (video 5) or KO (video 6) osteoclasts. Time is indicated as min:sec and frame is $16 \mu \mathrm{m}$. 


\section{REFERENCES}

Bhattacharya, R., and Cabral, F. (2004). A ubiquitous beta-tubulin disrupts microtubule assembly and inhibits cell proliferation. Mol. Biol. Cell 15, 31233131. doi: 10.1091/mbc.E04-01-0060

Bhattacharya, R., and Cabral, F. (2009). Molecular basis for class V beta-tubulin effects on microtubule assembly and paclitaxel resistance. J. Biol. Chem. 284, 13023-13032. doi: 10.1074/jbc.M900167200

Bhattacharya, R., Yang, H., and Cabral, F. (2011). Class V $\beta$-tubulin alters dynamic instability and stimulates microtubule detachment from centrosomes. Mol. Biol. Cell 22, 1025-1034. doi: 10.1091/mbc.E10-10-0822

Bhuwania, R., Castro-Castro, A., and Linder, S. (2014). Microtubule acetylation regulates dynamics of KIF1C-powered vesicles and contact of microtubule plus ends with podosomes. Eur. J. Cell Biol. 93, 424-437. doi: 10.1016/j.ejcb.2014.07. 006

Blangy, A., Bompard, G., Guerit, D., Marie, P., Maurin, J., Morel, A., et al. (2020). The osteoclast cytoskeleton - current understanding and therapeutic perspectives for osteoporosis. J. Cell Sci. 133:jcs244798. doi: 10.1242/jcs.244798

Bompard, G., van Dijk, J., Cau, J., Lannay, Y., Marcellin, G., Lawera, A., et al. (2018). CSAP acts as a regulator of TTLL-mediated microtubule glutamylation. Cell Rep. 25, 2866-2877. doi: 10.1016/j.celrep.2018.10.095

Brazier, H., Pawlak, G., Vives, V., and Blangy, A. (2009). The Rho GTPase Wrch1 regulates osteoclast precursor adhesion and migration. Int. J. Biochem. Cell Biol. 41, 1391-1401. doi: 10.1016/j.biocel.2008.12.007

Cervero, P., Wiesner, C., Bouissou, A., Poincloux, R., and Linder, S. (2018). Lymphocyte-specific protein 1 regulates mechanosensory oscillation of podosomes and actin isoform-based actomyosin symmetry breaking. Nat. Commun. 9:515. doi: 10.1038/s41467-018-02904-x

Cox, J., and Mann, M. (2008). MaxQuant enables high peptide identification rates, individualized p.p.b.-range mass accuracies and proteome-wide protein quantification. Nat. Biotechnol. 26, 1367-1372. doi: 10.1038/nbt.1511

Destaing, O., Saltel, F., Gilquin, B., Chabadel, A., Khochbin, S., Ory, S., et al. (2005). A novel Rho-mDia2-HDAC6 pathway controls podosome patterning through microtubule acetylation in osteoclasts. J. Cell Sci. 118, 2901-2911. doi: $10.1242 /$ jcs. 02425

Dogterom, M., and Koenderink, G. H. (2019). Actin-microtubule crosstalk in cell biology. Nat. Rev. Mol. Cell Biol. 20, 38-54. doi: 10.1038/s41580-018-0067-1

Gil-Henn, H., Destaing, O., Sims, N. A., Aoki, K., Alles, N., Neff, L., et al. (2007). Defective microtubule-dependent podosome organization in osteoclasts leads to increased bone density in Pyk2(-/-) mice. J. Cell Biol. 178, 1053-1064.

Guérit, D., Marie, P., Morel, A., Maurin, J., Verollet, C., Raynaud-Messina, B., et al. (2020). Primary myeloid cell proteomics and transcriptomics: importance of $\beta$-tubulin isotypes for osteoclast function. J. Cell Sci. 133:jcs239772. doi: $10.1242 /$ jcs. 239772

Guimbal, S., Morel, A., Guérit, D., Chardon, M., Blangy, A., and Vives, V. (2019). Dock5 is a new regulator of microtubule dynamic instability in osteoclasts. Biol. Cell 111:21. doi: 10.1111/boc.201900014

Ito, Y., Teitelbaum, S. L., Zou, W., Zheng, Y., Johnson, J. F., Chappel, J., et al. (2010). Cdc42 regulates bone modeling and remodeling in mice by modulating RANKL/M-CSF signaling and osteoclast polarization. J. Clin. Invest. 120, 19811993. doi: 10.1172/JCI39650

Janke, C., and Magiera, M. M. (2020). The tubulin code and its role in controlling microtubule properties and functions. Nat. Rev. Mol. Cell Biol. 21, 307-326. doi: 10.1038/s41580-020-0214-3

Kato, A., Naiki-Ito, A., Naitoh, I., Hayashi, K., Nakazawa, T., Shimizu, S., et al. (2018). The absence of class III $\beta$-tubulin is predictive of a favorable response to nab-paclitaxel and gemcitabine in patients with unresectable pancreatic ductal adenocarcinoma. Hum. Pathol. 74, 92-98. doi: 10.1016/j.humpath.2018.01.009

Khosla, S., and Hofbauer, L. C. (2017). Osteoporosis treatment: recent developments and ongoing challenges. Lancet Diabetes Endocrinol. 5, 898-907. doi: 10.1016/S2213-8587(17)30188-2

Koeppel, M. A., McCarthy, C. C., Moertl, E., and Jakobi, R. (2004). Identification and Characterization of PS-GAP as a novel regulator of caspase-activated PAK-2. J. Biol. Chem. 279, 53653-53664. doi: 10.1074/jbc.M410530200

Kopp, P., Lammers, R., Aepfelbacher, M., Woehlke, G., Rudel, T., Machuy, N., et al. (2006). The kinesin KIF1C and microtubule plus ends regulate podosome dynamics in macrophages. Mol. Biol. Cell 17, 2811-2823.
Leandro-García, L. J., Leskelä, S., Landa, I., Montero-Conde, C., ópez-Jiménez, E. L., Letón, R., et al. (2010). Tumoral and tissue-specific expression of the major human beta-tubulin isotypes. Cytoskeleton 67, 214-223. doi: 10.1002/cm.2 0436

Livak, K. J., and Schmittgen, T. D. (2001). Analysis of relative gene expression data using real-time quantitative PCR and the 2(-Delta Delta C(T)) Method. Methods 25, 402-408. doi: 10.1006/meth.2001.1262

Maurin, J., Morel, A., Hassen-Khodja, C., Vives, V., Jurdic, P., Machuca-Gayet, I., et al. (2018). Combined strategy of siRNA and osteoclast actin cytoskeleton automated imaging to identify novel regulators of bone resorption shows a nonmitotic function for anillin. Eur. J. Cell Biol. 97, 568-579. doi: 10.1016/j.ejcb. 2018.10.002

McMichael, B. K., Scherer, K. F., Franklin, N. C., and Lee, B. S. (2014). The RhoGAP activity of myosin IXB is critical for osteoclast podosome patterning, motility, and resorptive capacity. PLoS One 9:e87402. doi: 10.1371/journal.pone.0087402

Mounier, L., Morel, A., Ferrandez, Y., Morko, J., Vääräniemi, J., Gilardone, M., et al. (2020). Novel 2,7-diazaspiro[4,4]nonane derivatives to inhibit mouse and human osteoclast activities and prevent bone loss in ovariectomized mice without affecting bone formation. J. Med. Chem. 63, 13680-13694. doi: 10.1021/ acs.jmedchem.0c01201

O’Hagan, R., Silva, M., Nguyen, K. C. Q., Zhang, W., Bellotti, S., Ramadan, Y. H., et al. (2017). Glutamylation regulates transport, specializes function, and sculpts the structure of cilia. Curr. Biol. 27, 3430-3441. doi: 10.1016/j.cub.2017.09.066

Okumura, S., Mizoguchi, T., Sato, N., Yamaki, M., Kobayashi, Y., Yamauchi, H., et al. (2006). Coordination of microtubules and the actin cytoskeleton is important in osteoclast function, but calcitonin disrupts sealing zones without affecting microtubule networks. Bone 39, 684-693. doi: 10.1016/j.bone.2006.04. 010

Pamula, M. C., Ti, S.-C., and Kapoor, T. M. (2016). The structured core of human $\beta$ tubulin confers isotype-specific polymerization properties. J. Cell Biol. 213, 425-433. doi: 10.1083/jcb.201603050

Portran, D., Schaedel, L., Xu, Z., Théry, M., and Nachury, M. V. (2017). Tubulin acetylation protects long-lived microtubules against mechanical ageing. Nat. Cell Biol. 19, 391-398. doi: 10.1038/ncb3481

Raynaud, F., Homburger, V., Seveno, M., Vigy, O., Moutin, E., Fagni, L., et al. (2018). SNAP23-Kif5 complex controls mGlu1 receptor trafficking. J. Mol. Cell Biol. 10, 423-436. doi: 10.1093/jmcb/mjy031

Ren, X.-R., Du, Q.-S., Huang, Y.-Z., Ao, S.-Z., Mei, L., and Xiong, W.-C. (2001). Regulation of Cdc42 gtpase by proline-rich tyrosine kinase 2 interacting with Psgap, a novel pleckstrin homology and src homology 3 domain containing rhogap protein. J. Cell Biol. 152, 971-984. doi: 10.1083/jcb.152.5.971

Sanjana, N. E., Shalem, O., and Zhang, F. (2014). Improved vectors and genomewide libraries for CRISPR screening. Nat. Methods. 11, 783-784. doi: 10.1038/ nmeth.3047

Schäringer, K., Maxeiner, S., Schalla, C., Rütten, S., Zenke, M., and Sechi, A. (2021). LSP1-myosin1e bimolecular complex regulates focal adhesion dynamics and cell migration. FASEB J 35:e21268. doi: 10.1096/fj.202000740RR

Sekiguchi, M., Sobue, A., Kushima, I., Wang, C., Arioka, Y., Kato, H., et al. (2020). ARHGAP10, which encodes Rho GTPase-activating protein 10, is a novel gene for schizophrenia risk. Transl. Psychiatry 10:247. doi: 10.1038/s41398-02000917-z

Søe, K., and Delaissé, J.-M. (2017). Time-lapse reveals that osteoclasts can move across the bone surface while resorbing. J. Cell. Sci. 130, 2026-2035. doi: 10. $1242 /$ jcs. 202036

Spano, A. J., and Frankfurter, A. (2010). Characterization of anti-beta-tubulin antibodies. Methods Cell Biol. 95, 33-46. doi: 10.1016/S0091-679X(10)95003-6

Steenblock, C., Heckel, T., Czupalla, C., Espírito Santo, A. I., Niehage, C., Sztacho, M., et al. (2014). The Cdc42 guanine nucleotide exchange factor FGD6 coordinates cell polarity and endosomal membrane recycling in osteoclasts. J. Biol. Chem. 289, 18347-18359. doi: 10.1074/jbc.M113.504894

Ti, S.-C., Alushin, G. M., and Kapoor, T. M. (2018). Human $\beta$-tubulin isotypes can regulate microtubule protofilament number and stability. Dev. Cell 47, 175-190. doi: 10.1016/j.devcel.2018.08.014

Touaitahuata, H., Morel, A., Urbach, S., Mateos-Langerak, J., de Rossi, S., and Blangy, A. (2016). Tensin 3 is a new partner of Dock 5 that controls osteoclast podosome organization and activity. J. Cell Sci. 129, 3449-3461. doi: 10.1242/ jcs. 184622 
Tyanova, S., Temu, T., Sinitcyn, P., Carlson, A., Hein, M. Y., Geiger, T., et al. (2016). The perseus computational platform for comprehensive analysis of (prote)omics data. Nat. Methods 13, 731-740. doi: 10.1038/nmeth. 3901

Vemu, A., Atherton, J., Spector, J. O., Moores, C. A., and Roll-Mecak, A. (2017). Tubulin isoform composition tunes microtubule dynamics. Mol. Biol. Cell 28, 3564-3572. doi: 10.1091/mbc.E17-02-0124

Vemu, A., Atherton, J., Spector, J. O., Szyk, A., Moores, C. A., and Roll-Mecak, A. (2016). Structure and dynamics of single-isoform recombinant neuronal human tubulin. J. Biol. Chem. 291, 12907-12915. doi: 10.1074/jbc.C116.73 1133

Vives, V., Laurin, M., Cres, G., Larrousse, P., Morichaud, Z., Noel, D., et al. (2011). The Racl exchange factor Dock 5 is essential for bone resorption by osteoclasts. J. Bone Miner. Res. 26, 1099-1110. doi: 10.1002/jbmr.282

Xu, Z., Schaedel, L., Portran, D., Aguilar, A., Gaillard, J., Marinkovich, M. P., et al. (2017). Microtubules acquire resistance from mechanical breakage through intralumenal acetylation. Science 356, 328-332. doi: 10.1126/science. aai 8764

Zalli, D., Neff, L., Nagano, K., Shin, N. Y., Witke, W., Gori, F., et al. (2016). The actin-binding protein cofilin and its interaction with cortactin are required for podosome patterning in osteoclasts and bone resorption in vivo and in vitro. J. Bone Miner. Res. 31, 1701-1712. doi: 10.1002/jbmr.2851

Conflict of Interest: The authors declare that the research was conducted in the absence of any commercial or financial relationships that could be construed as a potential conflict of interest.

Publisher's Note: All claims expressed in this article are solely those of the authors and do not necessarily represent those of their affiliated organizations, or those of the publisher, the editors and the reviewers. Any product that may be evaluated in this article, or claim that may be made by its manufacturer, is not guaranteed or endorsed by the publisher.

Copyright (c) 2021 Maurin, Morel, Guérit, Cau, Urbach, Blangy and Bompard. This is an open-access article distributed under the terms of the Creative Commons Attribution License (CC BY). The use, distribution or reproduction in other forums is permitted, provided the original author(s) and the copyright owner(s) are credited and that the original publication in this journal is cited, in accordance with accepted academic practice. No use, distribution or reproduction is permitted which does not comply with these terms. 